\title{
SCIENTIFIC REPORTS

\section{Correlation-based network analysis combined with machine learning techniques highlight the role of the GABA shunt in Brachypodium sylvaticum freezing tolerance}

\author{
David Toubiana ${ }^{1,5}$, Nir Sade ${ }^{1,2,5}$, Lifeng Liu ${ }^{3}$, Maria del Mar Rubio Wilhelmi ${ }^{1}$, Yariv Brotman ${ }^{4}$,
} Urszula Luzarowska ${ }^{4}$, John P. Vogel ${ }^{3}$ \& Eduardo Blumwald ${ }^{1 *}$

Perennial grasses will account for approximately 16 billion gallons of renewable fuels by the year 2022 , contributing significantly to carbon and nitrogen sequestration. However, perennial grasses productivity can be limited by severe freezing conditions in some geographical areas, although these risks could decrease with the advance of climate warming, the possibility of unpredictable early cold events cannot be discarded. We conducted a study on the model perennial grass Brachypodium sylvaticum to investigate the molecular mechanisms that contribute to cold and freezing adaption. The study was performed on two different $B$. sylvaticum accessions, Ain1 and Osl1, typical to warm and cold climates, respectively. Both accessions were grown under controlled conditions with subsequent cold acclimation followed by freezing stress. For each treatment a set of morphological parameters, transcription, metabolite, and lipid profiles were measured. State-of-the-art algorithms were employed to analyze cross-component relationships. Phenotypic analysis revealed higher adaption of OsI1 to freezing stress. Our analysis highlighted the differential regulation of the TCA cycle and the GABA shunt between Ain 1 and Osl1. Osl1 adapted to freezing stress by repressing the GABA shunt activity, avoiding the detrimental reduction in fatty acid biosynthesis and the concomitant detrimental effects on membrane integrity.

The increase in energy demand coupled with the environmental impact of fossil fuels has led to the expansion of programs using perennial species as an alternative source of energy. In this context, perennial grasses, traditionally used as forage and turf, have become the preferred candidates for biomass cropping systems attributed to their ability to grow under low input conditions and high interception of sunlight ${ }^{1}$. Predictions showed that perennial grasses will account for approximately 16 billion gallons of renewable fuels by the year $2022^{2,3}$. Perennial grasses have shown to support carbon $(\mathrm{C})$ and nitrogen $(\mathrm{N})$ sequestration in the soil ${ }^{4-6}$, contributing to mitigate climate change and environmental degradation ${ }^{7-9}$. Brachypodium sylvaticum is a perennial grass with a simple genome, self-fertility, a short life cycle, and low growth requirements. Its recently released genome, expression gene atlas ${ }^{10}$ and well established genetic transformation have made it an efficient model to study the genetic, molecular, and physiological components of perennial grasses ${ }^{11,12}$.

Unfavorable winter conditions, including freezing, limit the geographical range of many important crops and reduce their productivity due to growth repression ${ }^{13,14}$. Low temperatures combined with high light intensity provoke the inhibition of the photosystem II and ROS production leading to oxidative stress ${ }^{15}$. In addition, ice formation in the apoplast induces both, cell membrane integrity stress and osmotic stress ${ }^{16}$. The plant's response to low temperatures involves extensive reprograming of gene expression via transcription factors ${ }^{17}$, resulting in concerted shifts of the metabolic network. Moreover, plants respond to cold stress by increasing the degree of

${ }^{1}$ Department of Plant Sciences, University of California, Davis, California, USA. ${ }^{2}$ School of Plant Sciences and Food Security, Tel Aviv University, Tel Aviv, Israel. ${ }^{3}$ DOE Joint Genome Institute, 2800 Mitchell Dr., Walnut Creek, CA, 94598, USA. 'Department of Life Sciences, Ben Gurion University of the Negev, Beer Sheva, Israel. ${ }^{5}$ These authors contributed equally: David Toubiana and Nir Sade. *email: eblumwald@ucdavis.edu 


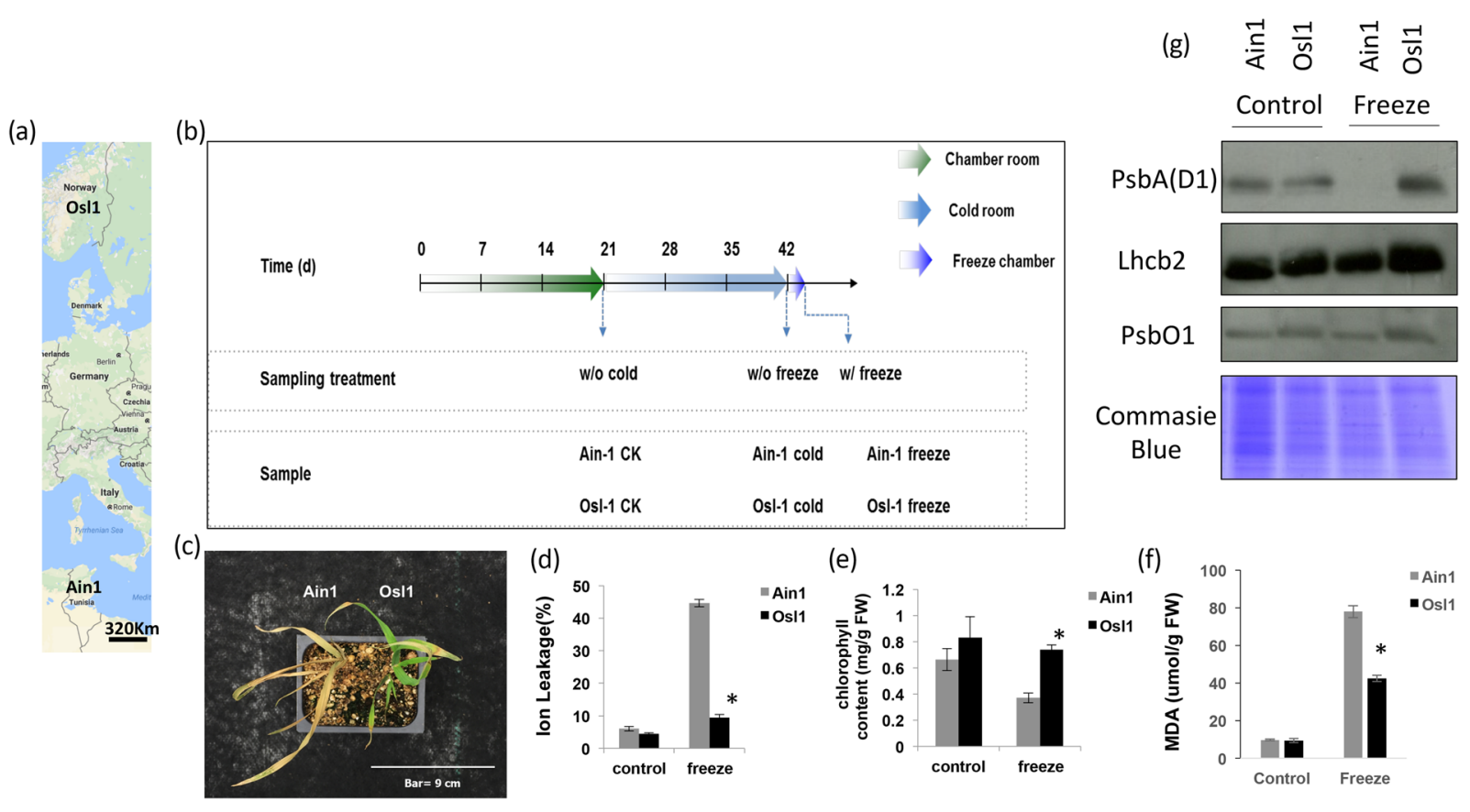

Figure 1. Effect of freezing on two B. Sylvaticum accessions (Ain1 and Osl1). (a) Geographical habitat of the B. Sylvaticum accessions (b) Experimental setup (c) Ain1 and Osl1 phenotype post-stress (d) Ain1 and Osl1 Ion leakage and (e) Chlorophyll content (f) Lipid Peroxidation (MDA) and (g) Immunoblot analysis of PSII subunits Lhcb2, PsbA (D1), and PsbO1. Values are Mean \pm SE $(n=3-12)$. The data was analyzed using Student's $\mathrm{t}$ test. Asterisks indicate significant differences $(\mathrm{p} \leq 0.05)$.

unsaturation of the membrane lipids and by remodeling lipids, which in turn may help the plant to avoid membrane integrity damage and achieve chilling tolerance ${ }^{18}$. The molecular and biochemical pathways regulating winter-hardiness in perennial grasses are poorly understood and deserve further attention.

With the advent of high-throughput platforms in the biological sciences, data driven analysis has become a key element for the integration of biological data of different backgrounds. A plethora of tools have been developed which tackle the complex problem of data integration from different perspectives, e.g. ${ }^{19-24}$. The biological interpretation of the data, however, is always study specific. Many of these tools make use of graph theory and network analysis, in one way or another, as the integration of heterogenic data is accomplished with ease and graph theory is readily equipped with a myriad of analytical and interpretational tools ${ }^{25-27}$. Correlation-based network analysis (CNA) is based on mathematically defined (dis)similarity measures that correlate different components to each other and the resulting correlation coefficients reflect the magnitude of the co-linear relationship of the components. In complex biological data, with thousands of different components assessed at various data points, the correlation is synonymous with coordinated behavior. With the exploration of the structural properties of graphs, CNA has been successfully used to postulate biological hypotheses which have been verified a posteriori ${ }^{28}$. Recently, a novel approach that combines CNA with machine learning $(\mathrm{ML})$ techniques, was used to predict the presence of metabolic pathways in tomato correlation networks $(\mathrm{CN})^{29}$.

Here, we investigated the response of two Brachypodium sylvaticum accessions (Osl1 and Ain1) to cold and freezing stress. Accession Osl1 is endemic to Northern Europe, while Ain 1 can be predominantly found in Northern Africa (Fig. 1a). We studied traits of the two accessions known to exert different phenotypes. Furthermore, we applied a top-down based approach integrating gene expression data and metabolic and lipid profiles from high throughput platforms and state-of-the-art algorithms for the analysis of big biological data. We employed the aforementioned 'CNA combined with machine learning techniques' approach for a comparative analysis between two correlation networks to identify genotype-specific 'active' metabolic pathways. Furthermore, we modified a recently developed genetic search algorithm ${ }^{30}$ to integrate gene expression data with metabolic profiles and detect candidate genes that may be key regulators for the identified 'active' metabolic pathways.

\section{Results}

Phenotypic analysis reveals Osl1 freezing tolerance. To morphologically characterize the effect of freezing stress on B. sylvaticum, freezing experiments were performed on two different B. sylvaticum accessions, Osl1 and Ain1 (Fig. 1a). Plants of both accessions were grown under controlled conditions for 21 days before being cold acclimated for additional 21 days. Subsequently, plants were moved into a freezing chamber for a freezing challenge (Fig. 1b). Seedling leaf samples were taken (i) before cold acclimation, (ii) at cold acclimation, and (iii) during the freezing challenge (Fig. 1b). After the freezing challenge, Ain 1 plants were severely damaged showing leaf necrosis while Osl1 remained viable (Fig. 1c). Freezing treatment led to increased ion leakage in both accessions as compared to the non-stressed control samples. However, the increase was more significant for Ain1 


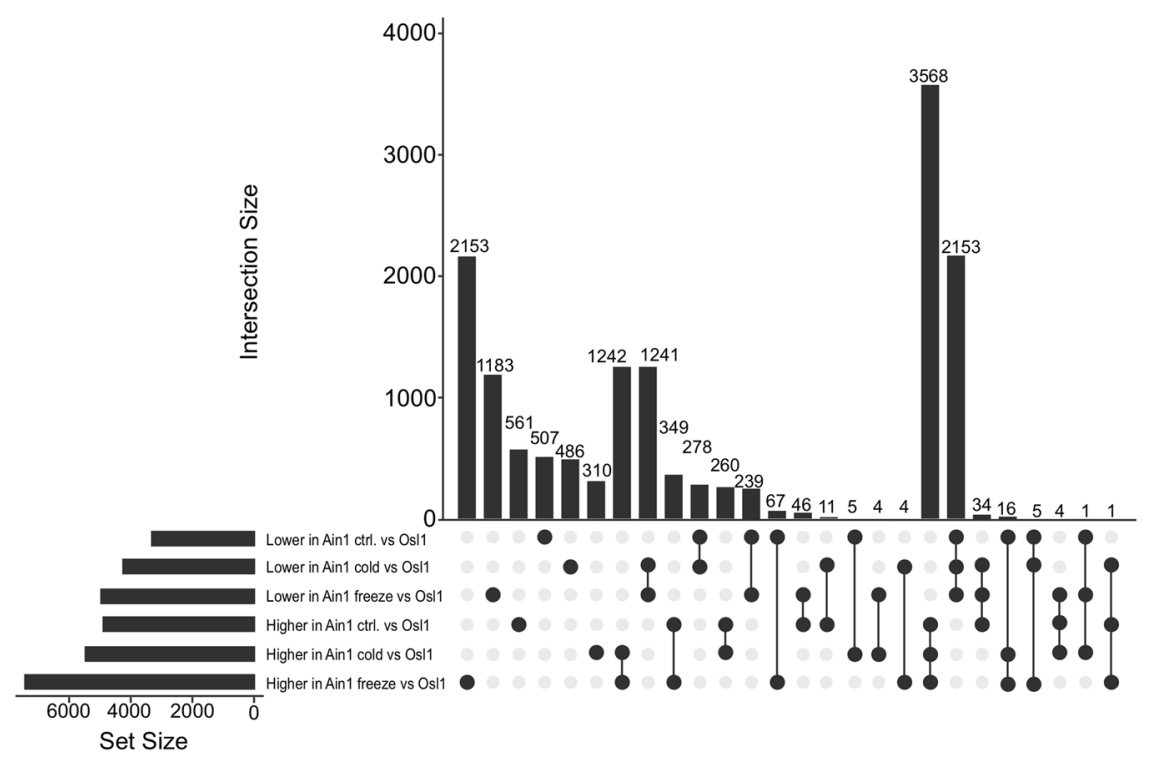

Figure 2. Intersections among stress differentially expressed genes across accessions and treatments. Number above bars indicates the number of genes within each intersection. Set size indicates the total number of significant genes in the treatments.

as compared to Osl1 (Fig. 1d). Chlorophyll content significantly decreased in Ain1 in response to the freezing treatment, while Osl1 kept higher chlorophyll content compared to Ain1 (Fig. 1e). To test for lipid peroxidation, MDA content was measured. The analysis showed that Ain1 plants displayed higher lipid peroxidation than Osl1 plants (Fig. 1f). Upon exposure to freezing conditions, the degradation of PSII subunits (D1, PsbO1, and Lhcb2) was more evident in Ain 1 plants as compared to Osl1 plants (Fig. 1g). Taken together, our results showed that Osl1 had a higher freezing-tolerance than Ain1 accession.

Differential gene expression analysis highlights freezing tolerance of Osl1. To gain an insight into differential gene expression profiles between the two accessions and their respective treatments, cDNA libraries were prepared and sequenced via a Illumina Hiseq platform, followed by differential gene expression analysis ${ }^{31}$. In total, 14,727 differentially expressed genes (DEGs) were identified when comparing the different treatments of Ain 1 and Osl1 samples (Fig. 2 - for a full list of genes IDs see Supplementary Data S1). The comparison of Ain1 control vs. Osl1 control samples identified 561 up-regulated DEGs and 517 down-regulated DEGs. The comparison between Ain 1 cold vs. Osl1 cold samples identified 310 up-regulated DEGs and 486 down-regulated DEGs. The comparison between Ain1 vs. Osl1 samples during freezing identified 2,153 up-regulated DEGs and 1,183 down-regulated DEGs.

In summary, the differential gene expression analysis demonstrated that the majority of up-regulated DEGs were specific to Ain 1 samples as compared to Osl1 samples, i.e. among the 5,721 unique DEGs resulting from the comparison between Ain 1 and Osl1 treatments, 3,568 were up-regulated in Ain1 while 2,153 were up-regulated in Osl1. In particular, the freezing treatment exerted the greatest impact on gene expression in Ain 1 supporting the notion of cold adaptation of the Osll ecotype.

Metabolite and lipid profiles are treatment specific. In an effort to assess metabolic changes between the accessions and their respective treatments, metabolite profiles were generated using a GC-MS platform as well as lipid profiles using an LC-MS platform. In total, 139 metabolites (Supplementary Fig. 1) and 195 lipids (Supplementary Fig. 2) were unequivocally identified. One-factorial analysis of variance revealed significant differences for 17 metabolites (Fig. 3) and 53 lipids (Supplementary Fig. 3a-c); i.e. two out of six diacylglycerols, six out of 22 digalactosyl-diacylglycerols, eight of 19 monogalactosyldiacylglycerols, five out of 28 phosphatidylcholines, four out of 15 phosphatidylethanolamines, eight out of 21 phosphatidylglycerols, one out of five ${ }^{32}$ phosphatidylinositols, four out of 15 phosphatidylserines, six out of 12 sulfoquinovosyldiacylglycerols, and six out of 52 triacylglycerides.

Posthoc Tukey test showed that sample means of metabolites (Fig. 3) and lipids (Fig. 4 and Supplementary Fig. $3 \mathrm{a}-\mathrm{c}$ ) were accession- and treatment-specific, e.g. for erythritol two significantly different means were identified - the lower mean level incorporating Ain 1 control, cold, freezing, and Osl1 cold (Fig. 3 - Tukey test group a) and the significant different higher mean containing Osl1 control and freezing samples (Fig. 3 - Tukey test group b). GABA revealed a decrease from control conditions to cold conditions in both accessions followed by a significant increase during freezing conditions. Galactinol showed higher levels in Osll and a general increase between control to freezing conditions in both accessions. Higher levels of erythritol and galactinol in the freezing tolerant accession supported the notion of sugar alcohols acting as agents against oxidative and osmotic stress ${ }^{32,33}$. Hydroxyproline and ornithine were also higher in Osl1 than in Ain 1 . While hydroxoproline is an essential element of the cell wall glycoprotein $\mathrm{RSH}^{34}$, elevated levels of ornithine have shown to alleviate the plant from abiotic 


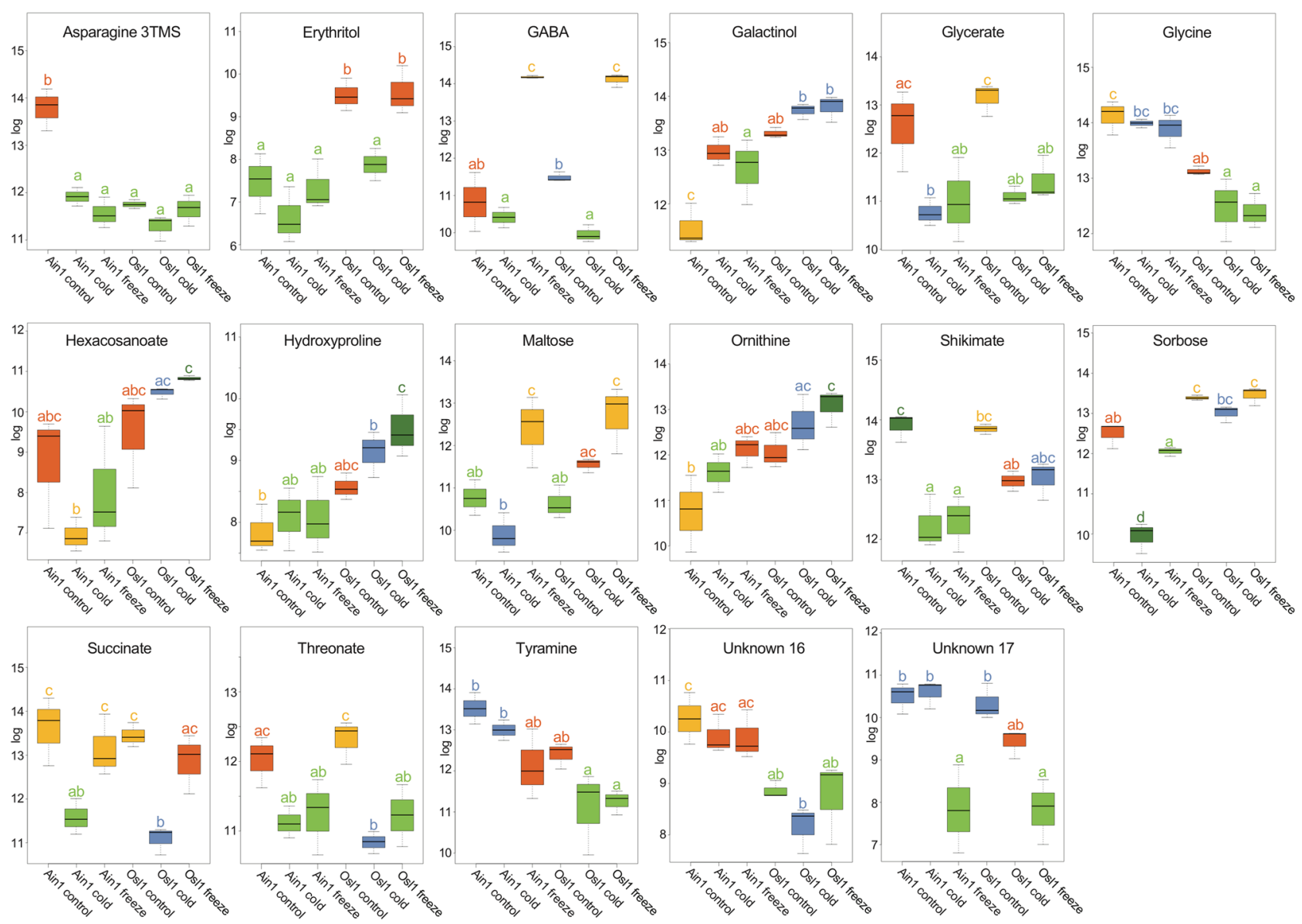

Figure 3. Significantly changing metabolites. Illustrated are the levels of the 17 significantly changing metabolites identified via analysis of variance in two Brachypodium sylvaticum accessions across different temperature treatments. X-axes represent the log of relative content, $y$-axes represent different accessions and their respective treatments. Values are the $\log ($ mean $) \pm S E(n=3)$. Different letters and color codes indicate significant differences $(\mathrm{p} \leq 0.05)$ according to posthoc Tukey's test.

stresses $^{35}$. Glycine and tyramine, on the other hand, showed generally higher means levels for Ain 1 samples and a decrease between control to freezing conditions in both accessions. The sugar maltose showed increased levels during freezing conditions in both accessions, while shikimate demonstrated a steep decrease during cold and freezing stress (in both accessions). The observation of metabolite specificity to accession and treatments supports the notion of the intertwined metabolic network, where metabolites are involved in many different metabolic pathways regulating various cellular aspects ${ }^{36,37}$.

For the 53 significantly changing lipids repetitive patterns were detected for the sulfoquinovosyldiacylglycerols (SQDG) and the triacylglycerides (TAG) (Fig. 4). For the SQDGs elevated levels were observed during Osl1 frozen, while for almost all frozen Ain 1 a steep decrease was visible in comparison to Ain 1 cold. The TAGs demonstrated an upward trend between Ain 1 control versus frozen Ain 1 and Osl1 control versus frozen Osl1. The finding of higher levels of SQDGs and TAGs during Osl1 freezing conditions was suggestive of their essential role for conserving membrane integrity and preventing ion leakage during cold and freezing ${ }^{38}$.

PCA emphasizes differences between Osl1 and Ain1 accessions. For the subsequent analysis, the metabolite and lipid profiles were combined into one single dataset (from here on onwards we will refer to the combined dataset as the compounds dataset). Principal component analysis (PCA) was applied to the 500 most variable genes of the normalized gene expression dataset ${ }^{31}$ and to the entire compounds dataset. For both PCAs, principal component (PC) 1 accounted for more than $90 \%$ of the variation observed (Fig. 5), while PCs2 accounted for $3.6 \%$ and $2.79 \%$ of the observed variation. For the PCA of the gene expression a clear separation between accessions was visible on PC1, while PC2 demonstrated a separation between treatments (Fig. 5a). The PCA based on the compound profiles revealed a gradual transition between samples on PC1 (Fig. 5b) reflecting the specificity of metabolites across treatments, as observed during the analysis for metabolic changes (see above). The loadings of PC1 revealed that while mainly metabolites could be identified on the negative end, solely lipids dominated the positive end, indicating a negative correlation between lipids and metabolites. On PC2 of the compound PCA (Fig. 5b), on the other hand, a separation between the accessions Ain 1 and Osl1 was visible. Here, 

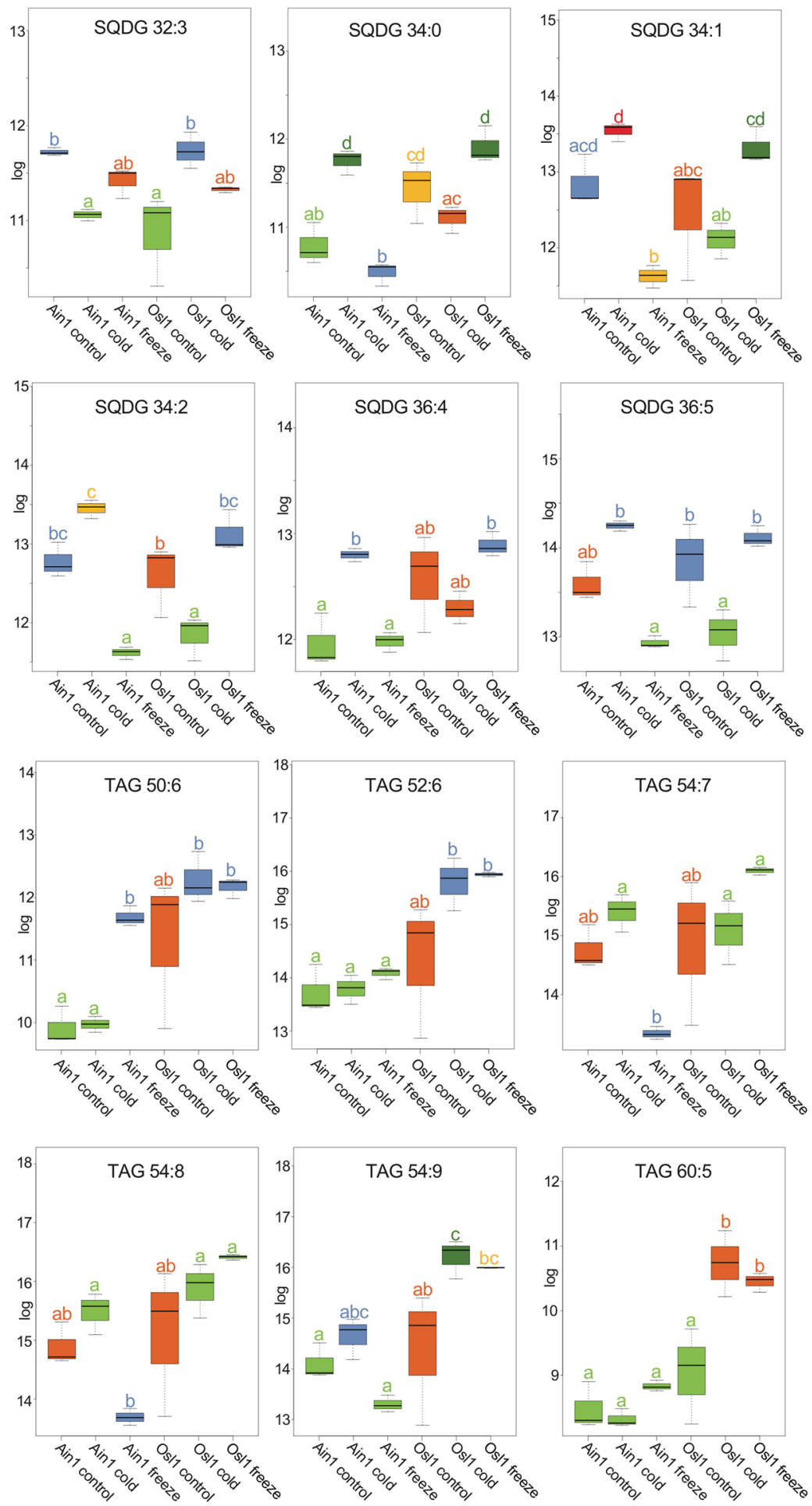

Figure 4. Significantly changing SQDG and TAG lipids. Illustrated are the levels of the eight significantly changing sulfoquinovosyldiacylglycerols (SQDG) and the triacylglycerides (TAG) identified via analysis of variance in two Brachypodium sylvaticum accessions across different temperature treatments. $\mathrm{X}$-axes represent the $\log$ of relative content, $y$-axes represent different accessions and their respective treatments. Values are the $\log (\operatorname{mean}) \pm \operatorname{SE}(n=3)$. Different letters and color codes indicate significant differences $(\mathrm{p} \leq 0.05)$ according to posthoc Tukey’s test.

the positive loadings were also dominated by the lipids, but several metabolites could be detected amongst them. Particularly TAGs and hexacosanoate, ornithine, GABA, proline, hydroxyproline, erythritol, galactinol, and melibiose recorded similar loadings, indicative of a positive correlation between them. 

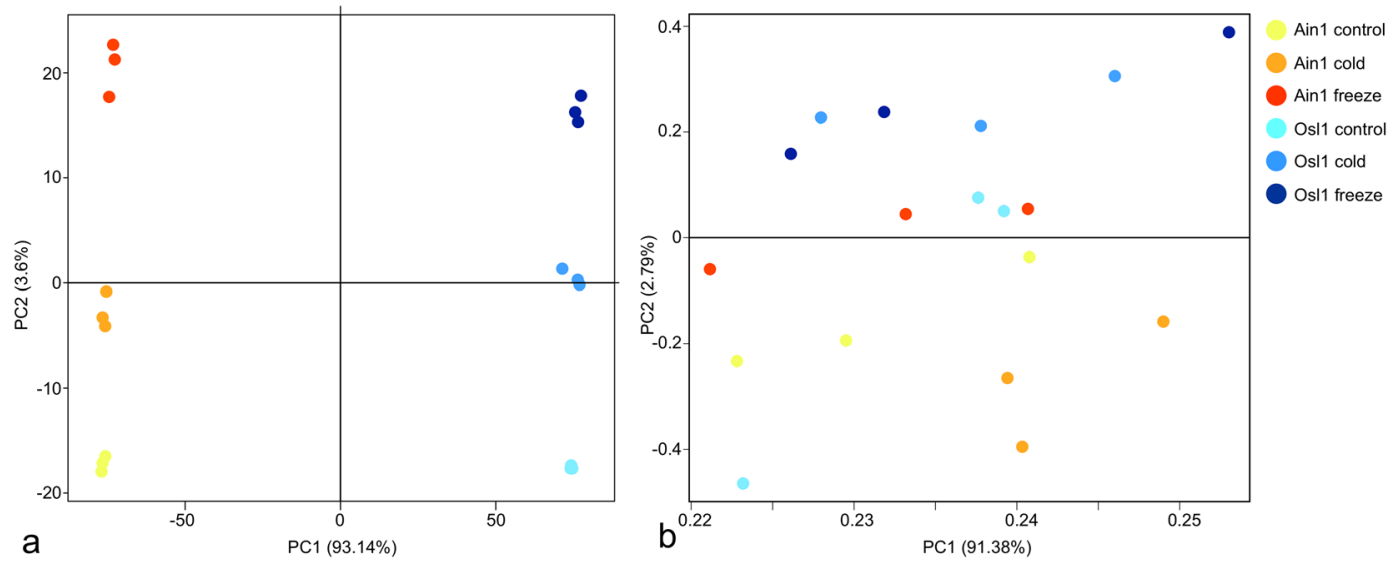

Figure 5. Principal component analysis (PCA) of gene expression and metabolite profiles. (a) PCA plot of Brachypodium sylvaticum accessions samples according to their normalized gene counts after variancestabilizing transformation. The first (PC1) and second (PC2) principal components are illustrated. Samples correspond to three biological replicates from two accessions (Ain1 and Osl1) and three different temperature regimes were analyzed - represented by different color-codes; (b) PCA plot of metabolite profiles of corresponding samples shown in (a).

Correlation-based network analysis emphasizes functional group of lipids in Osl1. Following the separation derived from the PCA, a correlation-based network (CN) was constructed for each Ain1 and Osl1 compounds datasets ${ }^{39}$. In the $\mathrm{CN}$, the nodes represent compounds and the edges between them the significant correlation coefficients $(r)$. Thresholds for the Ain 1 and Osll CNs were set to $r \geq 0.8$ and a q-value $\leq 0.05$ to remove spurious correlations. The Ain $1 \mathrm{CN}$ was composed of 334 nodes, corresponding to the compounds in the dataset, and 4,538 edges, of which 3,995 corresponded to positive correlations and 543 to negative correlations, resulting in a positive edges to negative edges (pe/ne) ratio of 7.36. The Osl1 CN contained 334 nodes and 6,317 edges, of which 6,230 corresponded to positive correlations and 87 to negative correlations, resulting in a pe/ ne ratio of 71.6. Due to the greater number of edges in the Osl1 network, it exerted greater values for the edge density (Ain $1=0.08$, Osl1 $=0.11$ ) and the average weighted degree of the network ( $\operatorname{Ain} 1=24.24$, Osl1 $=33.53$ ). Clustering of metabolites in a CN into communities may identify functional groups of nodes of similar chemical properties or groups of metabolites that are tightly connected due to shared regulatory processes ${ }^{26,39,40}$. Nodes in the network were clustered together using the walktrap community detecting algorithm ${ }^{41}$, revealing four lipid communities and one community containing metabolites in the Ain1 network (Fig. 6a) and two lipid communities and three metabolite communities in the Osl1 network (Fig. 6b). Of the TAG's identified via statistical analysis and PCA, in the Ain1 network TAG's 50:6, 54:7, 58:8, and 54:9 clustered into community 1, while TAG's 52:6 and 60:5 clustered into community 4. In the Osl1 network, on the other hand, all significant TAG's clustered into a single community, namely community 1 .

Among the lipid communities a small number of non-lipids were incorporated, mainly sugars, amino acids, fatty acids, and unknowns. To identify commonalities and differences between the networks, difference (Fig. 7a,b), intersection and symmetric difference networks (Supplementary Fig. 4a,b) were constructed. The analysis showed that 1,670 edges were common to both networks (Supplementary Fig. 4b), 2,868 out of the 4,538 edges were specific to the Ain1 network (Fig. 7a) and 4,647 out of the 6,317 edges were specific to the Osl1 network (Fig. 7b). Moreover, among all of the metabolites integrated into the lipid communities, only GABA appeared in both networks (Supplementary Fig. 4b), while altrose, maltose, sorbose, sucrose, docosanoate, octacosanoate, asparagine, and three unknowns were specific to the Ain1 network and raffinose, histidine, glutamine, hexacosanoate, and two unknowns were specific to the Osl1 network. Oligosaccharides and polysaccharides contribute to the stability of phospholipidic mono- and bilayers via their inclusion between polar headgroup $\mathrm{s}^{42}$. However, while the sugars associated with the Ain 1 network have shown to be a stabilizing agent for the plasma membrane during cold stress $^{43}$, raffinose (Osl1 network) appeared to be restrained to the chloroplast inner membrane ${ }^{44}$. Nevertheless, raffinose has been demonstrated to act as a cryoprotectant in cold acclimated Arabidopsis ecotypes ${ }^{45}$. In the same study, a positive correlation between raffinose and glutamine was demonstrated. Asparagine, on the other hand, decreased during cold adaptation in the perennial ryegrass ${ }^{46}$. The occurrence of different long chain fatty acids in the Ain1 and Osl1 difference network may hint at the discerned regulation of lipid biosynthesis ${ }^{47}$.

Among the putative functional group of lipids in the Ain1 network, one positive significant connection between GABA and TAG 50:6 (correlation coefficient 0.92) and three negative significant correlations to TAG's 54:7, 54:8, 54:9 (correlation coefficient $<-0.8$ ) were present. Furthermore, maltose maintained two significant negative connections to TAGs 54:7 and 54:8 (correlation coefficient $<-0.8$ ). In the Osl1 network, the GABA and maltose significant correlations did not appear. However, galactinol displayed a significant positive correlation to TAG 60:5, while hexacosanoate displayed significant positive correlations to all TAG's (correlation coefficient $>0.85$ ).

CNA combined with machine learning predicts GABA shunt activities for Ain1. To put the metabolic and expression data into a biological context, we applied novel algorithms for the analysis of big biological 


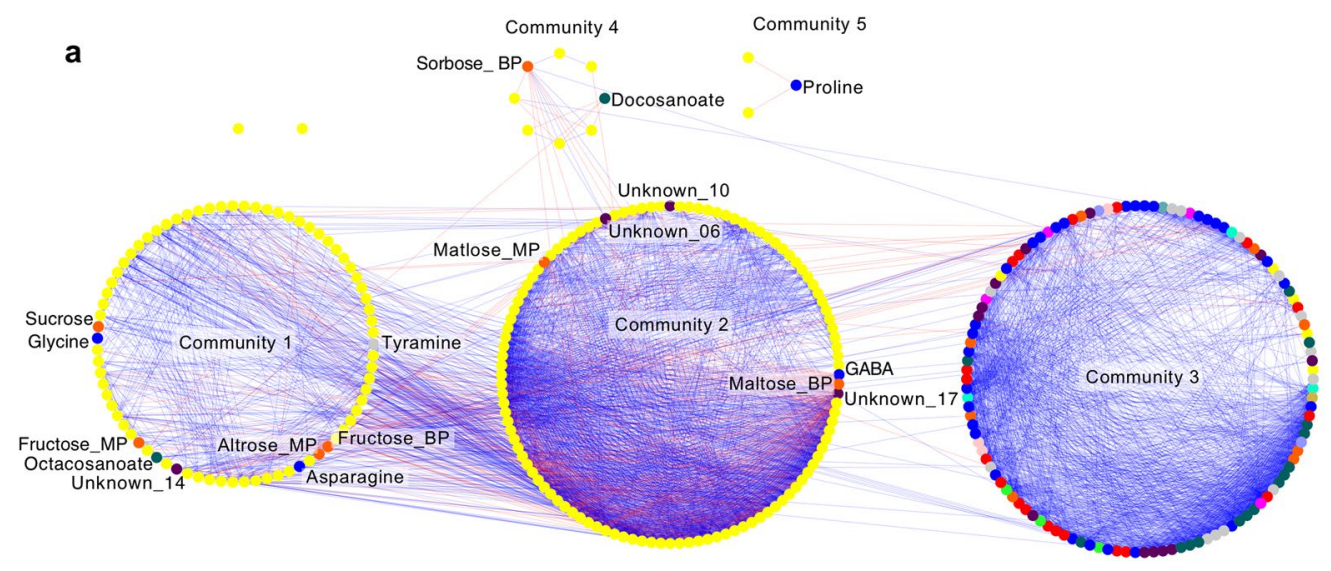

b


Figure 6. Correlation-based networks of Ain1 and Osl1. Network visualization of pairwise correlations of metabolites and lipids. The Pearson correlation was used to estimate correlation coefficients. Threshold tests for correlation coefficients $(r)$ and $p$-values were applied to detect significant correlations. Thresholds were set tor $r \geq 0.8$ and $q$ of $\leq 0.05$. Spurious correlations were removed, while significant correlations were transformed into network form. Metabolites are displayed as elliptical nodes and color-coded according to the compound classification; lipids are displayed as yellow elliptical nodes. Positive correlations are illustrated as blue edges, negative correlations as red edges. Communities in the networks were determined by the walktrap community detecting algorithm. (a) B. sylvaticum Ain1 correlation network; (b) B. sylvaticum Osl1 correlation network.

data. First, we applied CNA combined with ML techniques ${ }^{29}$. This approach explores the fact that metabolic pathways shape the topology of a CN. By mapping known metabolic pathways into the $\mathrm{CN}$ and learning their topological conformation, a ML model is generated capable of predicting the presence of unknown pathways. To do so, for each $\mathrm{CN}$ one ML model was generated (see above and Materials and Methods for details) employing the extreme gradient boost algorithm ${ }^{48}$. Performance evaluation of the ML models showed an AUC of the receiving operating characteristic curve of 0.986 for the Ain $1 \mathrm{CN}$ model and 0.992 for the Osl1 CN model (Supplementary Fig. 5). The confusion matrix of the models demonstrated an accuracy of 0.934 for the Ain 1 model and 0.921 for the Osl1 model (Table 1). The cross-validated models where then used to predict the presence/activity of 278 different metabolic pathways gathered from the PlantCyc repository ${ }^{36}$ at a prediction threshold value of $\geq 0.5$. Sensitivity analysis was applied to validate the prediction values of the metabolic pathways. Only pathways that tested positive during prediction and sensitivity analysis were considered positively predicted. The analysis suggested the presence of five pathways that occurred in Ain 1 and Osl1, 15 pathways specific to Ain1, and 19 pathways specific to Osl1 (Table 2). Pathways specific to Ain 1 centered mainly on the amino acids GABA, glutamate and the organic acids fumarate, succinate, 4-OH-benzoate, and the $\mathrm{N}$-compound putrescine, i.e. 4-aminobutanoate degradation I and IV, L-glutamate degradation IV, putrescine degradation IV, and the reductive TCA cycle I. TCA cycle intermediates, putrescine, glutamate, and GABA are inherently related, such that 2-oxoglutarate is converted via glutamate dehydrogenase (E.C. 1.4.1.3) to glutamate which in turn is decarboxylated via glutamate decarboxylase (E.C. 4.1.1.15) to GABA. Via the conversion of GABA to succinate semialdehyde mediated by 4 -aminobutyrate-aminotransferase (E.C. 2.6.19) and subsequently to succinate via succinate semialdehyde dehydrogenase (E.C. 1.2.1.24), the GABA shunt is completed. Moreover, GABA can be produced 


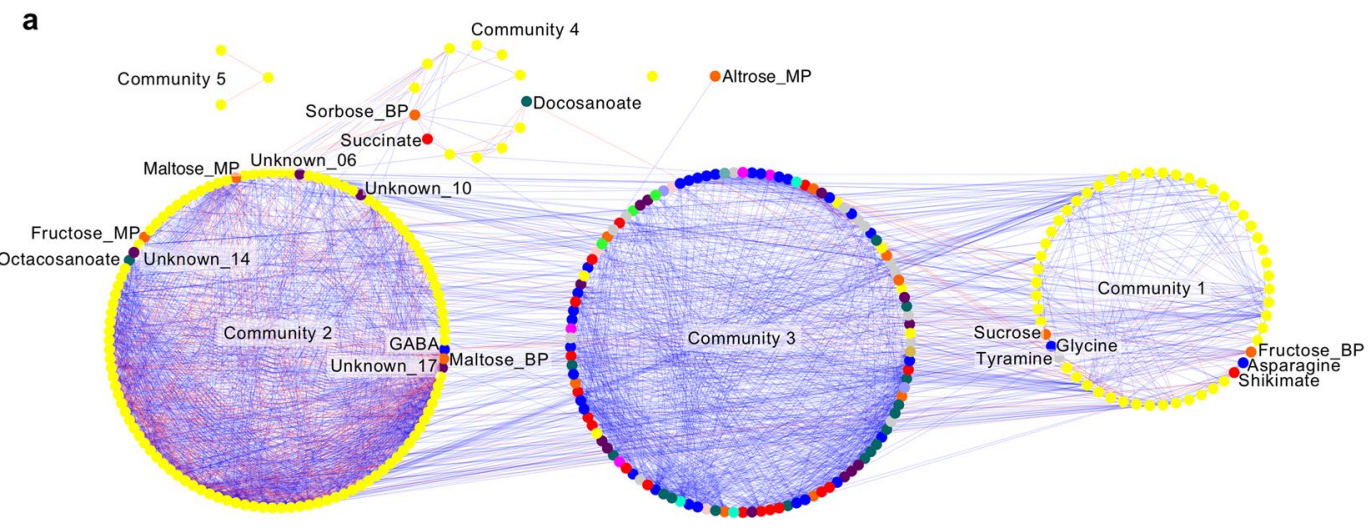

b

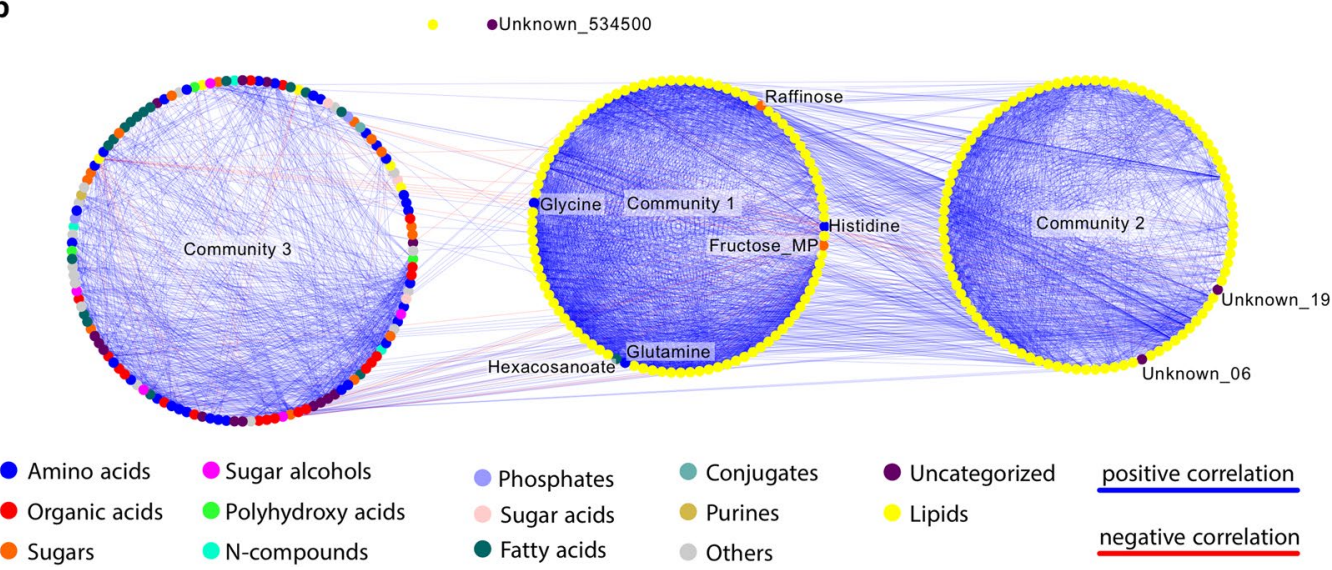

Figure 7. Difference set correlation-based networks of Ain 1 and Osl1. Network visualization of pairwise correlations of metabolites and lipids. The Pearson correlation was used to estimate correlation coefficients. Threshold tests for correlation coefficients $(r)$ and $p$-values were applied to detect significant correlations. Thresholds were set tor $r \geq 0.8$ and $q$ of $\leq 0.05$. Spurious correlations were removed, while significant correlations were transformed into network form. Differences between networks can be determined using set theory, were the differences sets highlight nodes and edges specific to a network. Metabolites are displayed as elliptical nodes and color-coded according to the compound classification; lipids are displayed as yellow elliptical nodes. Positive correlations are illustrated as blue edges, negative correlations as red edges. Communities in the networks were determined by the walktrap community-detecting algorithm. (a) $B$. sylvaticum Ain $1 \not \subset$ Osl1 difference correlation network; (b) B. sylvaticum Osl1 $\not \subset$ Ain 1 difference correlation network.

\begin{tabular}{|l|l|l|l|l|l|}
\hline \multirow{4}{*}{} & \multicolumn{2}{|l|}{ Ain1 ML model } & \multicolumn{2}{l|}{ Osl1 ML model } \\
\cline { 2 - 6 } & & \multicolumn{2}{|l|}{ Actual class } & \multicolumn{2}{l|}{ Actual class } \\
\hline \multirow{3}{*}{ Predicted class } & 1 & 2 & 1 & 2 \\
\cline { 2 - 6 } & 1 & 38 & 5 & 38 & 6 \\
\cline { 2 - 6 } & 2 & 0 & 33 & 0 & 32 \\
\hline
\end{tabular}

Table 1. Confusion Matrices of Ain 1 and Osl1 ML models. $1=$ positive instances, $2=$ negative instances.

using putrescine, which is oxidized by diamine oxidase (E.C. 1.4.3.22) to 4-amino-butanal and then catalyzed to GABA via aminobutyraldehyde dehydrogenase (E.C. 1.2.1.19). The pronounced occurrence of glutamate, GABA, putrescine and TCA cycle intermediates was indicative for the upregulation of the GABA shunt, which is known for sustaining the TCA cycle under abiotic and biotic stress conditions ${ }^{49-51}$. The relation between the GABA shunt and the TCA cycle was supported by identification of the reductive TCA cycle I pathway specific to Ain 1 (Table 2). Other noteworthy metabolic pathways detected for Ain 1 were free phenylpropanoid acid biosynthesis based on cis- and trans-sinnnapate and 4-OH-cinnamate, fatty acids biosynthesis based on malonate and adenosine-5-monophosphate, and photorespiration based on glycerate, glycerate-3-phosphate, glutamate, serine, glycine, and 2-oxoglutrate.

Pathways that were specific to Osl1, centered mainly on the amino acids asparagine, aspartate, cysteine, and tyrosine and the organic acid 2-oxo-glutarate, suggestive for ammonium and cyanide metabolism. Cyanogenic plants liberate cyanide from cyanogenic glucosides and lipids when they are in contact with predatory 


\begin{tabular}{|c|c|c|c|}
\hline & Metabolic pathways & Predictionvalue & $\begin{array}{l}\text { Sensitivity } \\
\text { analysis }\end{array}$ \\
\hline \multirow{5}{*}{ Ain $1+$ Osl1 } & gamma-glutamyl cycle & $0.74,0.96$ & $0.71,0.92$ \\
\hline & glutathione degradation & $0.74,0.96$ & $0.71,0.92$ \\
\hline & glycine biosynthesis & $0.74,0.74$ & $0.78,0.81$ \\
\hline & L-asparagine biosynthesis II & $0.74,0.85$ & $0.82,0.87$ \\
\hline & suberin monomers biosynthesis & $0.74,0.85$ & $0.78,0.85$ \\
\hline \multirow{15}{*}{ Ain 1} & 4-aminobutanoate degradation I & 0.96 & 0.94 \\
\hline & 4-aminobutanoate degradation IV & 0.96 & 0.94 \\
\hline & 4-hydroxybenzoate biosynthesis I (eukaryotes) & 0.74 & 0.85 \\
\hline & 4-hydroxybenzoate biosynthesis IV & 0.59 & 0.53 \\
\hline & 4-hydroxybenzoate biosynthesis $\mathrm{V}$ & 0.59 & 0.53 \\
\hline & fatty acid biosynthesis (plant mitochondria) & 0.59 & 0.56 \\
\hline & free phenylpropanoid acid biosynthesis & 0.59 & 0.50 \\
\hline & homocarnosine biosynthesis & 0.96 & 0.94 \\
\hline & L-glutamate degradation IV & 0.96 & 0.93 \\
\hline & nitric oxide biosynthesis II (mammals) & 0.93 & 0.92 \\
\hline & photorespiration & 0.74 & 0.78 \\
\hline & putrescine degradation IV & 0.74 & 0.78 \\
\hline & reductive TCA cycle I & 0.74 & 0.81 \\
\hline & trehalose degradation II (trehalase) & 0.93 & 0.90 \\
\hline & UMP biosynthesis II & 0.74 & 0.82 \\
\hline \multirow{19}{*}{ Osl1 } & (S)-reticuline biosynthesis I & 0.96 & 0.90 \\
\hline & 2'-deoxymugineic acid phytosiderophore biosynthesis & 0.73 & 0.74 \\
\hline & 3-cyano-L-alanine $+\mathrm{H}++2 \mathrm{H} 2 \mathrm{O}->$ ammonium $+\mathrm{L}$-aspartate & 0.73 & 0.76 \\
\hline & 4-hydroxyphenylpyruvate biosynthesis & 0.96 & 0.90 \\
\hline & ajugose biosynthesis II (galactinol-independent) & 0.88 & 0.80 \\
\hline & cyanide detoxification I & 0.96 & 0.91 \\
\hline & gamma-glutamyl cycle (plant pathway) & 0.73 & 0.70 \\
\hline & hypoglycin biosynthesis & 0.73 & 0.82 \\
\hline & L-asparagine biosynthesis I & 0.73 & 0.80 \\
\hline & L-asparagine degradation I & 0.73 & 0.76 \\
\hline & $\begin{array}{l}\text { L-glutamine }+\mathrm{L} \text {-aspartate }+\mathrm{ATP}+\mathrm{H} 2 \mathrm{O} \\
->\mathrm{L} \text {-glutamate }+\mathrm{L} \text {-asparagine }+\mathrm{AMP}+\text { diphosphate }+\mathrm{H}+\end{array}$ & 0.73 & 0.80 \\
\hline & L-phenylalanine biosynthesis III (cytosolic, plants) & 0.85 & 0.84 \\
\hline & L-tyrosine biosynthesis II & 0.96 & 0.90 \\
\hline & L-tyrosine biosynthesis III & 0.96 & 0.90 \\
\hline & rosmarinic acid biosynthesis I & 0.96 & 0.90 \\
\hline & rosmarinic acid biosynthesis II & 0.96 & 0.90 \\
\hline & S-methyl-5-thio-alpha-D-ribose 1-phosphate degradation & 0.73 & 0.79 \\
\hline & stachyose biosynthesis & 0.73 & 0.71 \\
\hline & superpathway of phospholipid biosynthesis II (plants) & 0.96 & 0.87 \\
\hline
\end{tabular}

Table 2. Prediction table.

herbivores ${ }^{52}$. Also, accumulation of hydrogen cyanide, as a co-product of ethylene biosynthesis, has been reported in sorghum and barley during abiotic stresses, such as water-deficit ${ }^{53}$ and salinity ${ }^{54}$. The accumulation of hydrogen cyanide in plants has been associated with cell signaling ${ }^{55}$ and nitrogen-recycling processes ${ }^{54}$. However, too high levels of hydrogen cyanide may result in plant death as for instance caused by bacteria ${ }^{56}$. In this regard, the occurrence of the cyanide detoxification I pathway in Osl1 may render beneficial to counteract cyanide contamination under freezing stress. Also, the superpathway of phospholipid biosynthesis based on serine and myo-inositol was predicted for Osl1. This pathway is one of the main producers of phospholipids for membranes in plants ${ }^{57}$ and may provide the means to sustain membrane integrity in Osl1. Other noteworthy pathways specific to Osl1 were stachyose biosynthesis based on galactinol, sucrose, raffinose and myo-inositol and ajugose biosynthesis based on raffinose and sucrose.

Genetic algorithm (GA) identifies accession-specific transcription factors and acetyltransferases. To integrate the gene expression data to the metabolic pathway analysis, we employed a GA that optimizes the correlation between a trait of interest and a subset of genes within a gene co-expression network, resulting in a number of genes that is sufficiently low to facilitate their analysis ${ }^{30}$. We made adequate modifications to the GA in order to correlate groups of metabolites representing the metabolic pathways identified in the previous 
analysis rather than single traits (see Materials and Methods). Correlations were estimated at absolute values so that negative correlating genes were included in the resulting set of genes. As a result of the previous analyses pointing to GABA and organic acids in relation to lipid regulation in Ain1, we investigated pathways associated with GABA metabolism (4-aminobutanoate degradation I and IV) and the TCA-cycle. For the metabolites associated with the 4 -aminobutanoate degradation I pathway, 74 genes were identified (Supplementary Data S2). The correlation coefficient between the first principal components of the metabolites and genes was computed with 0.698. The average expression of the 74 genes showed similar patterns in Ain 1 and Osl1 (Supplementary Fig. 6), revealing decreasing values from control to cold conditions followed by a significant increase under freezing. However, the greatest average values were achieved during Ain1 freezing, indicating increased transcription of genes associated with GABA, alanine, succinate, metabolism. A similar picture arose for 4-aminobutanoate degradation VI (GABA, alanine, succinate, pyruvate, 2-oxoglurate, and glutamate), where 108 genes were identified with a correlation coefficient of 0.796 . For the L-glutamate degradation IV pathway (2-oxo-glutrate, alanine, glutamate, GABA, pyruvate, and succinate), 104 genes were detected with a correlation coefficient of 0.619 . For the putrescine degradation IV pathway (putrescine and GABA), 112 genes were found with a correlation coefficient of 0.979 . Metabolites associated with the reductive TCA cycle 1 (citrate, fumarate, succinate, pyruvate, adenosine-5-monophosphate, 2-oxoglutrate) were also analyzed. Here, 461 genes were detected and the correlation coefficient was estimated at 0.859 . The average expression patterns demonstrated a similar pattern as before (Supplementary Fig. 6). Examination of the identified genes, highlighted two acetyltransferases of the Gcn5-related N-acetyltransferases family (GNAT) ${ }^{58}, 10$ transcription factor-associated genes, and 14 genes encoding membrane proteins. GNAT enzymes catalyze the transfer of an acyl moiety from acyl coenzyme A (acyl-CoA) to a large number of different substrates and are essential for lipid biosynthesis ${ }^{59}$. Here, the two acetyltransferases (Brasy3G100400 and Brasy9G171000 - Fig. 8) showed increased transcription in Osl1 and particularly in Osl1 under freezing. Out of the 10 transcription factor-associated genes, seven genes displayed Ain1- or Osl1-specific expression patterns. Brasy1G190400 (TCP family transcription factor), Brasy3G151900 (transcription factor HY5), Brasy4G087400 (BHLH transcription factor), Brasy6G010100 (MYB transcription factor) were, in general, more abundant in Ain1 and increased during freezing conditions, while Brasy4G312600, Brasy4G395200 (MYB family transcription factors) and Brasy5G38300 (RF2a transcription factor) were more abundant in Osl1 (Fig. 8). The distinct patterns of transcription associated with GABA and TCA-cycle intermediate metabolism and with transcription factors and acetyltransferases hinted at accession-specific regulation of lipid biosynthesis.

\section{Discussion}

We characterized the responses of two B. sylvaticum accessions, isolated from two geographical areas with contrasting climates (i.e., Osl1 from North Europe and Ain1 from North Africa - Fig. 1). Our aim was to characterize freezing tolerance mechanism(s) in this model perennial grass that may contribute into unraveling similar mechanisms in biomass crops such as switchgrass and Miscanthus ${ }^{2,60}$. Osl1 plants displayed higher freezing tolerance than Ain1, as indicated by increased vigor, higher chlorophyll contents, lower MDA, lower ion leakage and higher PSII protein accumulation (Fig. 1).

Next, we profiled all samples for gene expression and metabolites and lipids (compounds dataset). PC1 of the gene expression PCA showed a clear separation between accessions and separation of treatments on PC2 (Fig. 5a). The PCA of the compounds dataset showed a transitional change between treatments on PC1 and a separation between accessions on PC2 (Fig. 5b). The conclusions of the PCA were twofold: i) the differences observed on a transcriptional and metabolic level were mainly due to genotypic differences ii) posttranscriptional and posttranslational events were highly regulated, resulting in transitional rather than separational metabolic phenotypes between Ain 1 and Osl1. Differential gene expression analysis revealed increased changes of gene expression in Ain 1 in comparison to the freezing adapted Osl1. A lower degree of adjustment of transcription supported the notion of Osl1 adaptability to freezing stress by showing lower levels of response ${ }^{61,62}$. We constructed correlation-based networks for Ain 1 and Osl $1{ }^{39}$. Intersection, differences, and symmetric differences highlighted the topological differences between the networks (Figs. 6 and 7 and Supplementary Fig. 4). The higher network connectivity of the cold-adapted accession Osl1, together with a higher ratio ( $\sim 10$ fold $)$ of positive to negative correlations, supported the ability of Osl1 to adapt its metabolism at the initiation of the abiotic stress, similar to that observed in grapevine leaves ${ }^{63}$ or tomato seeds ${ }^{64}$.

Overwintering plants have evolved various strategies to minimize freezing injuries. These include the modification of membrane fatty acid composition, accumulation of carbohydrates and other compatible solutes and the production of antioxidants and antifreeze proteins $s^{65}$. Our statistical, multivariate, and correlation-based network analyses emphasized the role of GABA, glutamate, putrescine, TCA cycle intermediates, and SQDGs and TAGs lipids. While the link between GABA and the TCA cycle, and its involvement in abiotic stress, is well known ${ }^{66,67}$, we were particularly interested in their roles with respect to lipid biosynthesis and their potential impact on the membrane integrity of the two B. sylvaticum accessions. We employed state-of-the-art algorithms that place big biological data into biological context by combining CNA with machine-learning techniques. The idea behind this approach is that the computer learns from existing data. Based on recurring patterns that known metabolic pathways imprint onto correlation networks, the computer is able to predict the existence or 'activity' of unknown metabolic pathways. The approach has proven so powerful that it was able to predict the melibiose degradation pathway in tomato, although melibiose was not part of the original dataset ${ }^{29}$. Rooted on the two correlation-based networks, we generated two ML models (ML-Ain1 and ML-Osl1 - Supplementary Fig. 5) and then predicted the 'activity' of metabolic pathways as annotated in the PlantCyc collection ${ }^{36}$. While the ML-Ain 1 model was primarily associated with GABA metabolism and the reductive TCA cycle, these pathways were not predicted by the ML-Osl1 model (Table 2), indicative of their downregulation. We also employed a genetic algorithm that maximized the correlation between a subset of genes in a gene expression network and a trait of interest ${ }^{30}-$ in 

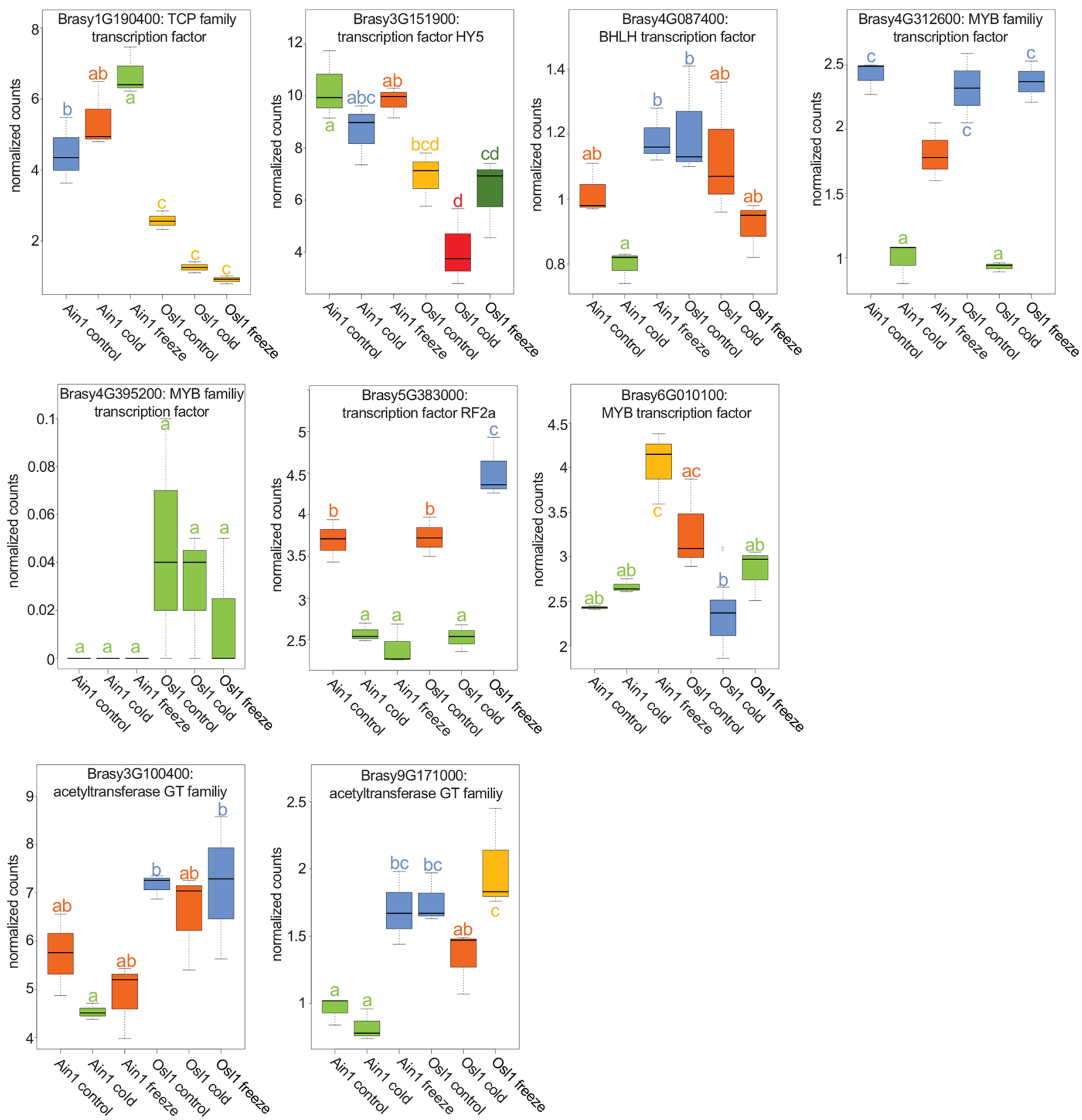

Figure 8. Transcription factors and acetyltransferases. Illustrated are the levels of the seven transcription factors activity associated genes (rows 1 and 2) and two acetyltransferases (row 3) identified via a genetic algorithm in two Brachypodium sylvaticum accessions across different temperature treatments. X-axes represent the normalized gene counts, $y$-axes represent different accessions and their respective treatments. Values are the mean $\pm \operatorname{SE}(n=3)$. Different letters and color codes indicate significant differences $(\mathrm{p} \leq 0.05)$ according to posthoc Tukey's test.

this case, sets of metabolites corresponding to metabolic pathways. The analysis highlighted a TCP family and the HY5-transcription factors showing elevated levels for Ain 1 in comparison to Osl. Also two acetyltransferases were identified with higher expression levels for Osl1 (Fig. 8).

The association between GABA and the TCA cycle intermediates is to replenish the TCA cycle via the GABA shunt and to maintain amino acid biosynthesis under low levels of energy ${ }^{49,50,67}$. GABA accumulation has been associated with abiotic stresses ${ }^{50,68}$. Increased GABA levels were shown in wheat and barley during freezing ${ }^{69}$. In our study, significant GABA increases were seen in both Ain 1 and Osl1 under freezing (Fig. 3). Nevertheless, we could predict GABA-associated pathways only in Ain1, indicating that under freezing Ain1 activated the GABA shunt, but not Osl1. Given the sensitivity of Ain1 to freezing, the activation of the GABA shunt may have had detrimental effects in Ain1. In transgenic Arabidopsis seeds the accumulation of high levels of GABA impaired fatty acid biosynthesis ${ }^{70}$. Bypassing the TCA cycle via the GABA shunt results in decreased levels of CoA moieties production, essential for fatty acid biosynthesis. Moreover, high levels of GABA have been associated with low 
levels of malate, which has been reported to be the preferred fatty acid elongation substrate $\mathrm{e}^{71,72}$. In yellow lupine seedlings, $\mathrm{C}$ atoms from acetate may be preferably incorporated into amino acid synthesis rather than fatty acid synthesis ${ }^{73}$ and similar mechanisms may play a role in reducing fatty acid biosynthesis leading to membrane leakage under freezing conditions in Ain1. In Osl1, on the other hand, the GABA shunt activity was downregulated as a result of normal TCA cycle activities and the maintenance of fatty acid biosynthesis, which are regulated by acetyltransferases, leading to the formation of functional, non-leaky membranes.

Taken together, our top-down-based approach, employing state-of-the-art algorithms for big data, was able to filter through physiological, transcription, metabolite and lipid data to provide evidence highlighting different regulatory mechanisms taken place in Ain 1 and Osl1 during freezing. While the upregulation of the GABA shunt activity showed detrimental effects on fatty acid biosynthesis, Osl1 downregulated the GABA shunt activity to counteract the inhibition of fatty acid biosynthesis. Consequently, membrane leakage was reduced contributing to the survival of Osl1 plants under freezing conditions.

\section{Methods}

Plant material, growth conditions, and experimental design. Seeds from B. sylvaticum accessions Ain 1 and Osl1 were obtained from the USDA National Plant Germplasm System (https://www.ars-grin.gov/ npgs/). For the cold experiments, the seeds were stratified in a cold room at $10 \mathrm{~h} / 1 \mathrm{~h}$ light $/$ dark cycles at $4{ }^{\circ} \mathrm{C}$ for 2 weeks before sown into soil. Germination and growth were achieved in a chamber room $16 \mathrm{~h} / 8 \mathrm{~h}$ light-dark cycles at $26^{\circ} \mathrm{C} / 18^{\circ} \mathrm{C}$, respectively, for 3 weeks. For cold acclimation, the plants were moved into a cold room at $10 \mathrm{~h} / 14 \mathrm{~h}$ light/dark cycles at $4^{\circ} \mathrm{C}$, for 3 weeks.

The freezing experiments were conducted in a temperature and light controlled growth chamber. The initial $24 \mathrm{~h}$ were composed of an $8 \mathrm{~h}$ light period at $4^{\circ} \mathrm{C}$, followed by a dark period of $2 \mathrm{~h}$ at $4{ }^{\circ} \mathrm{C}, 30 \mathrm{~min}$ at $2{ }^{\circ} \mathrm{C}, 30 \mathrm{~min}$ at $0^{\circ} \mathrm{C}, 4 \mathrm{~h}$ at $-2 \mathrm{C}, 8 \mathrm{~h}$ at $-5.5^{\circ} \mathrm{C}, 30 \mathrm{~min}$ at $-2^{\circ} \mathrm{C}$, and $30 \mathrm{~min}$ at $1^{\circ} \mathrm{C}$. For the subsequent $24 \mathrm{~h}$, plants were kept at dark at $4^{\circ} \mathrm{C}$. After the freezing treatment, all plants were moved to growth chambers where they were kept at $16 \mathrm{~h} / 8 \mathrm{~h}$ light-dark cycles at $26^{\circ} \mathrm{C} / 18^{\circ} \mathrm{C}$, respectively.

Leaf samples of seedlings were collected i) before the cold acclimation (CK) treatment was initiated, ii) before the freezing challenge was initiated, and iii) after $8 \mathrm{~h}$ of freezing at $-5.5^{\circ} \mathrm{C}$. All samples were subjected to RNA and metabolite extraction for subsequent gene expression and metabolomics analysis, respectively (see below). Ion leakage measurements and immunoblot analysis were performed immediately after freezing challenge using control plants as a reference control. The plant damage phenotyping and sampling for chlorophyll and MDA contents were performed after 2 weeks of recovery in growth chamber rooms using control plants as a reference control.

Electrolyte leakage. Shoot samples for electrolyte leakage were harvested immediately after freezing challenge. Electrolyte leakage was measured as described previously ${ }^{74}$.

Inmunoblot analysis. Plant leaf tissues were weighed, frozen in liquid $\mathrm{N}_{2}$, and ground in three volumes of $2 \times$ Laemmli sample buffer. Total proteins were separated by SDS-PAGE, transferred to a polyvinylidene difluoride membrane (Bio-Rad), and probed as described previously ${ }^{75}$. Antibodies raised against PsbA/D1 (AS01016), and Lhcb2 (AS01003) and PsbO 1 (AS05092), were obtained from Agrisera (Vannas, Sweden). Horseradish peroxidase-conjugated secondary antibodies were purchased from Santa Cruz Biotechnology.

Chlorophyll measurements. Shoots were grinded in liquid $\mathrm{N}_{2}$ and weighed. Chlorophyll was extracted in $80 \%$ acetone, and the absorbance was measured at $663 \mathrm{~nm}$ and $645 \mathrm{~nm}$ (Synergy ${ }^{\mathrm{TM}}$ Mx Microplate Reader; BioTek, USA). Total chlorophyll content was calculated as described elsewhere ${ }^{76}$.

Malondialdehyde measurements (MDA). Shoots were grinded in liquid $\mathrm{N}_{2}$ and weighed. Tissue was homogenized with $5 \mathrm{ml}$ of $50 \mathrm{mM} \mathrm{NaH} \mathrm{PO}_{4}-\mathrm{Na}_{2} \mathrm{HPO}_{4}$ buffer $\mathrm{pH} 7.5$ and centrifuged at $20,000 \mathrm{~g}$ for $25 \mathrm{~min}$. For MDA measurements, $4 \mathrm{ml}$ of $20 \%$ trichloroacetic acid containing $0.5 \%$ thiobarbituric acid were added to a $1 \mathrm{ml}$ aliquot of the supernatant. The mixture was heated at $95^{\circ} \mathrm{C}$ for $30 \mathrm{~min}$, quickly cooled in ice, and then centrifuged at $10,000 \mathrm{~g}$ for $10 \mathrm{~min}$. The absorbance of the supernatant was measured at $532 \mathrm{~nm}$ and $600 \mathrm{~nm}^{77}$.

Metabolite profiling by gas chromatography-mass spectrometry. Metabolites were extracted using the MTBE method as described elsewhere ${ }^{78}$. One hundred-fifty $\mu$ l vacuum-dried polar phases samples were derivatized and subjected to GC-MS analysis as described previously ${ }^{79}$. The GC-MS data were obtained using an Agilent 7683 series auto-sample (Agilent Technologies, http://www.home.agilent.com), coupled to an Agilent 6890 gas-chromatograph-Leco Pegasus two time-of-flight mass spectrometer (Leco: http://www.leco. $\mathrm{com} /$ ). Identical chromatogram acquisition parameters were applied to those previously used ${ }^{80}$. Chromatograms were exported from the LECO CHROMATOF software (version 3.34) to the R software. Ion extraction, peak detection, retention time alignment and library searching were obtained using the TargetSearch package from Bioconductor $^{81}$. The resulting data matrix was used for further analysis.

Lipid profiling. Lipids were extracted using the MTBE method as described previously ${ }^{77}$. Five hundred $\mu \mathrm{l}$ vacuum-dried organic phase samples were processed using ultra-performance liquid chromatography, on a C8 reverse phase column coupled with Fourier transform mass spectrometry (UPLC-FT-MS) (Thermo-Fisher, http://www.thermofisher.com) in positive and negative ionization modes. Processing of chromatograms, peak detection and integration were performed using REFINER MSH 10 (GeneData, http://www.genedata.com). Processing of mass spectrometry data included the removal of the fragmentation information, isotopic peaks and chemical noise. Selected features were annotated using an in-house lipid database ${ }^{80}$. 
Statistical analysis of metabolite and lipid dataset. All metabolite and lipid data was natural-log transformed. Missing values (4.4\%) were imputed using data imputation provided by Bioconductor (http://www. bioconductor.org/) package pcaMethods ${ }^{82}$. Analysis of variance (ANOVA) was run for each metabolite and lipid. To detect significant differences, $\mathrm{p}$-values (threshold $\leq 0.05$ ) were Bonferroni corrected to account for multiple hypothesis testing. Subsequently, a posthoc Tukey test was performed.

Library preparation and sequencing. RNA was extracted with Trizol (Invitrogen) and PureLink RNA Mini Kit (Ambion) and then treated with TURBO DNase (Ambion). The RNA quality control was accessed using 2100 Bioanalyzer and RNA 6000 Nano Kit (Agilent), Qubit RNA BR Assay Kit (Thermol Fisher Scientific) and Nanodrop (Thermol Fisher Scientific). RNA-sequencing libraries were constructed using TruSeq Stranded mRNA Library Preparation Kit (Illumina). PolyA + RNA was isolated from $1 \mu \mathrm{g}$ total RNA and then fragmented. The cDNA was synthesized, adenylated at $3^{\prime}$ ends and ligated with adaptors. The libraries were amplified with 8 cycles of polymerase chain reaction (PCR). Sequencing was done on a Illumina Hiseq platform of $2 \times 150 \mathrm{bp}$. Filtered reads from each library were aligned to the reference genome using HISAT version 0.1.4-beta ${ }^{83}$, featureCounts was used to generate the raw gene counts. Gene counts were normalized using DESeq. 2 (version 1.8.1) (11 $^{31}$ DESeq. 2 was also used to determine differentially expressed genes between pairs at a $q$-value of $\leq 0.05$.

PCA and correlation-based network analysis. Principal component analyses were performed on the metabolite combined with the lipid dataset as well as on the gene expression datasets to determine clusters of associated samples. For the identified clusters, metabolite correlation networks $(\mathrm{CN})$ were constructed as described before ${ }^{39}$. In brief, all metabolites were pairwise-correlated using Pearson correlation. Threshold tests for correlation coefficients $(r)$ and $p$-values were applied to detect significant correlations. The threshold for $r$-values was set to $\geq 0.8$ for the Ain 1 and Osl1 network, respectively. A false discovery rate ${ }^{84}$ was applied to adjust for multiple hypothesis testing at a $q$-value of $\leq 0.05$. Spurious correlations were removed, while significant correlations were transformed into network form.

Identification of metabolic pathways within CN's. Identification of metabolic pathways within CN's was performed essentially as described in ${ }^{29}$. The approach employs machine-learning techniques to train a model based on known metabolic pathways and then predicts the presence of pathways. The applied method uses pathways based on the PlantCyc repository (https://plantcyc.org//) ${ }^{36}$ However, a collection of metabolic pathways associated with $B$. sylvaticum has yet to be established. Therefore, for the current study, a consensus set of pathways found in all 101 plant species listed in PlantCyc version 13 was generated, detecting 38 consensus pathways (positive instances). The consensus pathways were mapped to the CN's upon which for each pathway a set of network features was computed $=$ positive training set ${ }^{29}$. As a negative training set 19 random pathways (negative instances) from MetaCyc collection (https://metacyc.org/) ${ }^{85}$ that could not be found within the PlantCyc collection but could be mapped to the CN's were selected. In addition, 19 random subsets of metabolites were generated accounting for the other half of the negative training set. Also here, for each instance of the negative training set the aforementioned network features were computed. To avoid over-fitting, the most important features were selected for each training-set. Next, the gradient boosting (xgboost) algorithm ${ }^{48}$ was employed with a 10 -fold cross validation to generate ML models for the Ain1 and Osl1 networks. Predictions for all test-set metabolic pathways were generated for the final models. The quality of ML models were assessed using performance measures, i.e. the area under the curve (AUC) of the receiving operating characteristic curve, which was generated by plotting the true positive rate against the false positive rate at different prediction thresholds. An AUC close to 1 indicated a perfect classifier, while an AUC of 0.5 indicated a poor classifier that could also be achieved by chance. Another mean to demonstrate ML performances is the usage of confusion matrices, where the predicted vs. the actual classification of positive and negative instances is captured in table form. The classifications of the predicted instances correspond to the rows, while the actual classifications correspond to the columns. Positive instances are denoted as ' 1 ' and negative instances are denoted as '2'. Derived from the confusion matrix is the accuracy measure, which is the proportion of correctly classified positive and negative instances. Finally, sensitivity analyses were performed based on the final models, where subsets (80\%) of the training-instances were randomly chosen to recreate models with identical settings. Sensitivity analyses were performed to test for the robustness of the ML model. After each model generation, the test-set instances were subjected to prediction. This analysis was performed with 100 repetitions, after which the average prediction values and their corresponding variance values were computed. Test-set pathways that demonstrated prediction values $\geq 0.5$ in the original model as well as in the average values of the sensitivity analysis were considered validly predicted.

Genetic algorithm. To identify genes associated with metabolic pathways, a genetic algorithm to optimize the correlation between a trait of interest and a subset of genes within a gene co-expression network trait to gene was used as described previously ${ }^{30}$. The genetic algorithm was adapted so that essentially:

$$
A R G M A X_{C}\{|\operatorname{cor}(\operatorname{prcomp}(\operatorname{Expr}[\cdot, C]), \operatorname{prcomp}(\operatorname{Pr}[\cdot, M]))|\}
$$

where $C$ is a subset of genes in the module, $\operatorname{Exp} r[\cdot, C]$ is the expression matrix narrowed down to $C, M$ is a subset of metabolites corresponding to the metabolic pathway, $\operatorname{Pr}[\cdot, M]$ is the metabolite profile matrix narrowed down to $M$, prcomp is the $1^{\text {st }}$ principal component of a matrix, and cor is the Pearson correlation.

\section{Data availability}

Data associated with the current study can be found under: https://figshare.com/account/home\#/projects/68999.

Received: 2 October 2019; Accepted: 14 February 2020;

Published online: 11 March 2020 


\section{References}

1. Dohleman, F. G., Heaton, E. A. \& Long, S. P. Perennial grasses as second-generation sustainable feedstocks without conflict with food production. Handbook of Bioenergy Economics and Policy, 27-37, https://doi.org/10.1007/978-1-4419-0369-3_3 (2010).

2. Carroll, A. \& Somerville, C. Cellulosic biofules. Annu. Rev. Plant Biol. 60, 165-182 (2009).

3. Houghton, J., Weatherwax, S. \& Ferrell, J. Breaking the Biological Barriers to Cellulosic Ethanol: A Joint Research Agenda. Report No. DOE/SC-00954055, (United States, 2006).

4. Yang, Y., Tilman, D., Furey, G. \& Lehman, C. Soil carbon sequestration accelerated by restoration of grassland biodiversity. Nat. Commun. 10, https://doi.org/10.1038/s41467-019-08636-w (2019).

5. Sumiyoshi, Y. et al. Belowground impacts of perennial grass cultivation for sustainable biofuel feedstock production in the tropics. GCB. Bioenergy 9, 694-709, https://doi.org/10.1111/gcbb.12379 (2017).

6. Sainju, U. M., Singh, H. P. \& Singh, B. P. Soil carbon and nitrogen in response to perennial bioenergy grass, cover crop and nitrogen fertilization. Pedosphere 27, 223-235, https://doi.org/10.1016/s1002-0160(17)60312-6 (2017).

7. Tilman, D., Hill, J. \& Lehman, C. Carbon-negative biofuels from low-input high-diversity grassland biomass. Science 314, 1598-1600, https://doi.org/10.1126/science.1133306 (2006).

8. Glover, J. D. et al. Harvested perennial grasslands provide ecological benchmarks for agricultural sustainability. Agr. Ecosyst. Environ. 137, 3-12, https://doi.org/10.1016/j.agee.2009.11.001 (2010).

9. Di Virgilio, N. et al. Four-year measurement of net ecosystem gas exchange of switchgrass in a Mediterranean climate after longterm arable land use. Gcb. Bioenergy 11, 466-482, https://doi.org/10.1111/gcbb.12523 (2019).

10. Gordon, S. P., Liu, L. \& Vogel, J. P. In Genetics and Genomics of Brachypodium. Plant Genetics and Genomics: Crops and Models Vol. 18 (ed. John P. Vogel) 313-325 (Springer, 2015).

11. Steinwand, M. A., Young, H. A., Bragg, J. N., Tobias, C. M. \& Vogel, J. P. Brachypodium sylvaticum, a Model for Perennial Grasses: Transformation and Inbred Line Development. Plos One 8, https://doi.org/10.1371/journal.pone.0075180 (2013).

12. Sade, N. et al. Salt tolerance of two perennial grass Brachypodium sylvaticum accessions. Plant Mol. Biol. 96, 305-314, https://doi. org/10.1007/s11103-017-0696-3 (2018).

13. Heidarvand, L. \& Amiri, R. M. What happens in plant molecular responses to cold stress? Acta Physiol. Plant. 32, 419-431, https:// doi.org/10.1007/s11738-009-0451-8 (2010).

14. Eremina, M., Rozhon, W. \& Poppenberger, B. Hormonal control of cold stress responses in plants. Cell. Mol. Life Sci. 73, 797-810, https://doi.org/10.1007/s00018-015-2089-6 (2016).

15. Takahashi, S. \& Murata, N. How do environmental stresses accelerate photoinhibition? Trends Plant Sci. 13, 178-182, https://doi. org/10.1016/j.tplants.2008.01.005 (2008).

16. Sandve, S. R. et al. Molecular mechanisms underlying frost tolerance in perennial grasses adapted to cold climates. Plant Sci. 180, 69-77, https://doi.org/10.1016/j.plantsci.2010.07.011 (2011).

17. Van Buskirk, H. A. \& Thomashow, M. F. Arabidopsis transcription factors regulating cold acclimation. Physiol. Plantarum 126, 72-80, https://doi.org/10.1111/j.1399-3054.2006.00625.x (2006).

18. Korn, M. et al. Predicting Arabidopsis Freezing Tolerance and Heterosis in Freezing Tolerance from Metabolite Composition. Mol. Plant 3, 224-235, https://doi.org/10.1093/mp/ssp105 (2010).

19. Jansen, R. et al. A Bayesian networks approach for predicting protein-protein interactions from genomic data. Science 302, 449-453, https://doi.org/10.1126/science.1087361 (2003).

20. Hwang, D. et al. A data integration methodology for systems biology. P. Natl. Acad. Sci. USA 102, 17296-17301, https://doi. org/10.1073/pnas.0508647102 (2005).

21. Myers, C. L. \& Troyanskaya, O. G. Context-sensitive data integration and prediction of biological networks. Bioinformatics 23, 2322-2330, https://doi.org/10.1093/bioinformatics/btm332 (2007).

22. Chung, S. Y. \& Wong, L. S. Kleisli: a new tool for data integration in biology. Trends Biotechnol. 17, 351-355, https://doi.org/10.1016/ s0167-7799(99)01342-6 (1999).

23. Letunic, I. et al. SMART 4.0: towards genomic data integration. Nucleic Acids Res. 32, D142-D144, https://doi.org/10.1093/nar/ gkh088 (2004).

24. von Mering, C. et al. STRING 7 - recent developments in the integration and prediction of protein interactions. Nucleic Acids Res. 35, D358-D362, https://doi.org/10.1093/nar/gk1825 (2007).

25. Newman, M. O. \& University Press, O., 2009). Networks: An Introduction. (Networks: An Introduction, 2009).

26. Newman, M. E. J. Communities, modules and large-scale structure in networks. Nat. Phys. 8 (2012).

27. Newman, M. E. J. \& Girvan, M. Finding and evaluating community structure in networks. Phys. Rev. E 69, https://doi.org/10.1103/ PhysRevE.69.026113 (2004).

28. Toubiana, D. et al. Combined correlation-based network and mQTL analyses efficiently identified loci for branched-chain amino acid, serine to threonine, and proline metabolism in tomato seeds. Plant J. 81, 121-133, https://doi.org/10.1111/tpj.12717 (2015).

29. Toubiana, D. et al. Combined network analysis and machine learning allows the prediction of metabolic pathways from tomato metabolomics data. Commun. Biol. 2, https://doi.org/10.1038/s42003-019-0440-4 (2019).

30. Toubiana, D., Puzis, R., Sadka, A. \& Blumwald, E. A Genetic Algorithm to Optimize Weighted Gene Co-Expression Network Analysis. J. Comput. Biol., https://doi.org/10.1089/cmb.2019.0221.

31. Love, M. I., Huber, W. \& Anders, S. Moderated estimation of fold change and dispersion for RNA-seq data with DESeq. 2. Genome Biol. 15, https://doi.org/10.1186/s13059-014-0550-8 (2014).

32. Keunen, E., Peshev, D., Vangronsveld, J., Van den Ende, W. \& Cuypers, A. Plant sugars are crucial players in the oxidative challenge during abiotic stress: extending the traditional concept. Plant Cell Environ. 36, 1242-1255, https://doi.org/10.1111/pce.12061 (2013).

33. Vinocur, B. \& Altman, A. Recent advances in engineering plant tolerance to abiotic stress: achievements and limitations. Curr. Opin. Biotech. 16, 123-132, https://doi.org/10.1016/j.copbio.2005.02.001 (2005).

34. Hall, Q. \& Cannon, M. C. The cell wall hydroxyproline-rich glycoprotein RSH is essential for normal embryo development in Arabidopsis. Plant Cell 14, 1161-1172, https://doi.org/10.1105/tpc.010477 (2002).

35. Kalamaki, M. S., Merkouropoulos, G. \& Kanellis, A. K. Can ornithine accumulation modulate abiotic stress tolerance in Arabidopsis? Plant Signaling Behav. 4, 1099-1101 (2009).

36. Schlapfer, P. et al. Genome-Wide Prediction of Metabolic Enzymes, Pathways, and Gene Clusters in Plants. Plant Physiol. 173, 2041-2059, https://doi.org/10.1104/pp.16.01942 (2017).

37. Kanehisa, M., Sato, Y., Furumichi, M., Morishima, K. \& Tanabe, M. New approach for understanding genome variations in KEGG. Nucleic Acids Res. 47, D590-D595, https://doi.org/10.1093/nar/gky962 (2019).

38. Barrero-Sicilia, C., Silvestre, S., Haslam, R. P. \& Michaelson, L. V. Lipid remodelling: Unravelling the response to cold stress in Arabidopsis and its extremophile relative Eutrema salsugineum. Plant Sci. 263, 194-200, https://doi.org/10.1016/j. plantsci.2017.07.017 (2017).

39. Toubiana, D., Fernie, A. R., Nikoloski, Z. \& Fait, A. Network analysis: tackling complex data to study plant metabolism. Trends Biotechnol. 31, 29-36, https://doi.org/10.1016/j.tibtech.2012.10.011 (2013).

40. Kose, F., Weckwerth, W., Linke, T. \& Fiehn, O. Visualizing plant metabolomic correlation networks using clique-metabolite matrices. Bioinformatics 17, 1198-1208, https://doi.org/10.1093/bioinformatics/17.12.1198 (2001).

41. Pons, P. \& Latapy, M. Computing communities in large networks using random walks. Lect. Notes Comput. Sc. 3733, 284-293 (2005). 
42. Vereyken, I. J., Chupin, V., Demel, R. A., Smeekens, S. C. M. \& De Kruijff, B. Fructans insert between the headgroups of phospholipids. BBA. Biomembranes 1510, 307-320, https://doi.org/10.1016/s0005-2736(00)00363-1 (2001).

43. Valluru, R., Lammens, W., Claupein, W. \& Van den Ende, W. Freezing tolerance by vesicle-mediated fructan transport. Trends Plant Sci. 13, 409-414, https://doi.org/10.1016/j.tplants.2008.05.008 (2008).

44. Nagele, T. \& Heyer, A. G. Approximating subcellular organisation of carbohydrate metabolism during cold acclimation in different natural accessions of Arabidopsis thaliana. New Phytol. 198, 777-787, https://doi.org/10.1111/nph.12201 (2013).

45. Klotke, J., Kopka, J., Gatzke, N. \& Heyer, A. G. Impact of soluble sugar concentrations on the acquisition of freezing tolerance in accessions of Arabidopsis thaliana with contrasting cold adaptation - evidence for a role of raffinose in cold acclimation. Plant Cell Environ. 27, 1395-1404, https://doi.org/10.1111/j.1365-3040.2004.01242.x (2004).

46. Draper, S. R. Amino-acid changes associated with low-temperature treatment of Lolium-perenne. Phytochemistry 11, 639-641, https://doi.org/10.1016/0031-9422(72)80025-6 (1972).

47. Ohlrogge, J. \& Browse, J. Lipid biosynthesis. Plant Cell 7, 957-970, https://doi.org/10.1105/tpc.7.7.957 (1995).

48. Friedman, J. H. Greedy function approximation: A gradient boosting machine. Annl. Stat. 29, 1189-1232, https://doi.org/10.1214/ aos/1013203451 (2001).

49. Bouche, N., Lacombe, B. \& Fromm, H. GABA signaling: a conserved and ubiquitous mechanism. Trends Cell Biol. 13, 607-610, https://doi.org/10.1016/j.tcb.2003.10.001 (2003).

50. Bouche, N. \& Fromm, H. GABA in plants: just a metabolite? Trends Plant Sci. 9, 110-115, https://doi.org/10.1016/j. tplants.2004.01.006 (2004).

51. Bown, A. W., MacGregor, K. B. \& Shelp, B. J. Gamma-aminobutyrate: defense against invertebrate pests? Trends Plant Sci. 11, 424-427, https://doi.org/10.1016/j.tplants.2006.07.002 (2006).

52. Gleadow, R. M. \& Woodrow, I. E. Constraints on effectiveness of cyanogenic glycosides in herbivore defense. J. Chem. Ecol. 28, 1301-1313, https://doi.org/10.1023/a:1016298100201 (2002).

53. O'Donnell, N. H. et al. Effects of PEG-induced osmotic stress on growth and dhurrin levels of forage sorghum. Plant Physiol. Bioch. 73, 83-92, https://doi.org/10.1016/j.plaphy.2013.09.001 (2013).

54. Maršálová, L., Vítámvás, P., Hynek, R., Práśil, I. T. \& Kosová, K. Proteomic Response of Hordeum vulgare cv. Tadmor and Hordeum marinum to Salinity Stress: Similarities and Differences between a Glycophyte and a Halophyte. Front. Plant Sci. 7, 1154, https://doi. org/10.3389/fpls.2016.01154 (2016).

55. Gotor, C. et al. Signaling by hydrogen sulfide and cyanide through post-translational modification. J. Exp. Bot. 70, 4251-4265, https://doi.org/10.1093/jxb/erz225 (2019).

56. Blom, D., Fabbri, C., Eberl, L. \& Weisskopf, L. Volatile-mediated killing of Arabidopsis thaliana by bacteria is mainly due to hydrogen cyanide. Appl. Environ. Microb. 77, 1000-1008, https://doi.org/10.1128/aem.01968-10 (2011).

57. Xu, C. C., Yu, B., Cornish, A. J., Froehlich, J. E. \& Benning, C. Phosphatidylglycerol biosynthesis in chloroplasts of Arabidopsis mutants deficient in acyl-ACP glycerol-3-phosphate acyltransferase. Plant J. 47, 296-309, https://doi. org/10.1111/j.1365-313X.2006.02790.x (2006).

58. Vetting, M. W. et al. Structure and functions of the GNAT superfamily of acetyltransferases. Arch. Biochem. Biophys. 433, 212-226, https://doi.org/10.1016/j.abb.2004.09.003 (2005).

59. Ud-Din, A., Tikhomirova, A. \& Roujeinikova, A. Structure and Functional Diversity of GCN5-Related N-Acetyltransferases (GNAT). Int. J. Mol. Sci. 17, https://doi.org/10.3390/ijms17071018 (2016).

60. Dohleman, F. G. \& Long, S. P. More Productive Than Maize in the Midwest: How Does Miscanthus Do It? Plant Physiol. 150, 2104-2115, https://doi.org/10.1104/pp.109.139162 (2009).

61. Begcy, K., Weigert, A., Egesa, A. O. \& Dresselhaus, T. Compared to australian cultivars, european summer wheat (triticum aestivum) overreacts when moderate heat stress is applied at the pollen development stage. Agronomy-Basel 8, https://doi.org/10.3390/ agronomy8070099 (2018).

62. Dresselhaus, T. \& Huckelhoven, R. Biotic and Abiotic Stress Responses in Crop Plants. Agron. (Basel) 8, https://doi.org/10.3390/ agronomy8110267 (2018).

63. Hochberg, U. et al. Metabolite profiling and network analysis reveal coordinated changes in grapevine water stress response. BMC Plant Biol. 13, https://doi.org/10.1186/1471-2229-13-184 (2013).

64. Rosental, L. et al. Environmental and genetic effects on tomato seed metabolic balance and its association with germination vigor. BMC Genomics 17, https://doi.org/10.1186/s12864-016-3376-9 (2016).

65. Thomashow, M. F. Plant cold acclimation: Freezing tolerance genes and regulatory mechanisms. Annu. Rev. Plant Phys. 50, 571-599, https://doi.org/10.1146/annurev.arplant.50.1.571 (1999).

66. Michaeli, S. et al. A mitochondrial GABA permease connects the GABA shunt and the TCA cycle, and is essential for normal carbon metabolism. Plant J. 67, 485-498, https://doi.org/10.1111/j.1365-313X.2011.04612.x (2011).

67. Fait, A., Fromm, H., Walter, D., Galili, G. \& Fernie, A. R. Highway or byway: the metabolic role of the GABA shunt in plants. Trends Plant Sci. 13, 14-19, https://doi.org/10.1016/j.tplants.2007.10.005 (2008).

68. Seifikalhor, M., Aliniaeifard, S., Hassani, B., Niknam, V. \& Lastochkina, O. Diverse role of gamma-aminobutyric acid in dynamic plant cell responses. Plant Cell Rep. 38, 847-867, https://doi.org/10.1007/s00299-019-02396-z (2019).

69. Mazzucotelli, E., Tartari, A., Cattivelli, L. \& Forlani, G. Metabolism of gamma-aminobutyric acid during cold acclimation and freezing and its relationship to frost tolerance in barley and wheat. J. Exp. Bot. 57, 3755-3766, https://doi.org/10.1093/jxb/erl141 (2006).

70. Fait, A. et al. Targeted Enhancement of Glutamate-to-gamma-Aminobutyrate Conversion in Arabidopsis Seeds Affects CarbonNitrogen Balance and Storage Reserves in a Development-Dependent Manner. Plant Physiol. 157, 1026-1042, https://doi. org/10.1104/pp.111.179986 (2011).

71. Smith, R. G., Gauthier, D. A., Dennis, D. T. \& Turpin, D. H. Malate-dependent and pyruvate-dependent fatty-acid synthesis in leukoplastids from developing castor endosperm. Plant Physiol. 98, 1233-1238, https://doi.org/10.1104/pp.98.4.1233 (1992).

72. Pleite, R., Pike, M. J., Garces, R., Martinez-Force, E. \& Rawsthorne, S. The sources of carbon and reducing power for fatty acid synthesis in the heterotrophic plastids of developing sunflower (Helianthus annuus L.) embryos. J. Exp. Bot. 56, 1297-1303, https:// doi.org/10.1093/jxb/eri130 (2005)

73. Borek, S., Ratajczak, W. \& Ratajczak, L. A transfer of carbon atoms from fatty acids to sugars and amino acids in yellow lupine (Lupinus luteus L.) seedlings. J. Plant Physiol. 160, 539-545, https://doi.org/10.1078/0176-1617-00763 (2003).

74. Sukumaran, N. P. \& Weiser, C. J. An excised leaflet test for evaluating potato frost tolerance. HortScience 7, 467-468 (1972).

75. Wang, S. \& Blumwald, E. Stress-induced chloroplast degradation in Arabidopsis is regulated via a process independent of autophagy and senescence-associated vacuoles. Plant Cell 26, 4875-4888, https://doi.org/10.1105/tpc.114.133116 (2014).

76. Porra, R. J. The chequered history of the development and use of simultaneous equations for the accurate determination of chlorophylls a and b. Photosynth. Res. 73, 149-156, https://doi.org/10.1023/a:1020470224740 (2002)

77. Giavalisco, P. et al. Elemental formula annotation of polar and lipophilic metabolites using C-13, N-15 and S-34 isotope labelling, in combination with high- resolution mass spectrometry. Plant J. 68, 364-376, https://doi.org/10.1111/j.1365-313X.2011.04682.x (2011).

78. Sade, N. et al. Delaying chloroplast turnover increases water-deficit stress tolerance through the enhancement of nitrogen assimilation in rice. J. Exp. Bot. 69, 867-878, https://doi.org/10.1093/jxb/erx247 (2018). 
79. Lisec, J., Schauer, N., Kopka, J., Willmitzer, L. \& Fernie, A. R. Gas chromatography mass spectrometry-based metabolite profiling in plants. Nat. Protoc. 1, 387-396, https://doi.org/10.1038/nprot.2006.59 (2006).

80. Caldana, C. et al. High-density kinetic analysis of the metabolomic and transcriptomic response of Arabidopsis to eight environmental conditions. Plant J. 67, 869-884, https://doi.org/10.1111/j.1365-313X.2011.04640.x (2011).

81. Cuadros-Inostroza, A. et al. TargetSearch - a Bioconductor package for the efficient preprocessing of GC-MS metabolite profiling data. BMC Bioinformatics 10, https://doi.org/10.1186/1471-2105-10-428 (2009).

82. Stacklies, W., Redestig, H., Scholz, M., Walther, D. \& Selbig, J. pcaMethods - a bioconductor package providing PCA methods for incomplete data. Bioinformatics 23, 1164-1167, https://doi.org/10.1093/bioinformatics/btm069 (2007).

83. Kim, D., Landmead, B. \& Salzberg, S. L. HISAT: a fast spliced aligner with low memory requirements. Nat. Methods 12, 357-U121, https://doi.org/10.1038/nmeth.3317 (2015).

84. Benjamini, Y. \& Hochberg, Y. Controlling the false discovery rate - a practical and powerful approach to multiple testing. J. Roy. Stat. Soc. B Met. 57, 289-300 (1995).

85. Caspi, R. et al. The MetaCyc database of metabolic pathways and enzymes. Nucleic Acids Res. 46, D633-D639, https://doi. org/10.1093/nar/gkx935 (2018).

\section{Acknowledgements}

This work was supported by the US Department of Energy, Office of Science, Office of Biological and Environmental Research under Award Number DE-SC0008797 and by the Will W. Lester Endowment from the University of California.

\section{Author contributions}

D.T. analyzed, designed and performed the in silico experiments. N.S., L.L., M.M.R.W., D.T., J.P.V. and E.B. designed and performed the in vivo experiments. U.L. and Y.B. performed metabolic and lipid profiling. D.T., N.S. and E.B. wrote the manuscript.

\section{Competing interests}

The authors declare no competing interests.

\section{Additional information}

Supplementary information is available for this paper at https://doi.org/10.1038/s41598-020-61081-4.

Correspondence and requests for materials should be addressed to E.B.

Reprints and permissions information is available at www.nature.com/reprints.

Publisher's note Springer Nature remains neutral with regard to jurisdictional claims in published maps and institutional affiliations.

Open Access This article is licensed under a Creative Commons Attribution 4.0 International License, which permits use, sharing, adaptation, distribution and reproduction in any medium or format, as long as you give appropriate credit to the original author(s) and the source, provide a link to the Creative Commons license, and indicate if changes were made. The images or other third party material in this article are included in the article's Creative Commons license, unless indicated otherwise in a credit line to the material. If material is not included in the article's Creative Commons license and your intended use is not permitted by statutory regulation or exceeds the permitted use, you will need to obtain permission directly from the copyright holder. To view a copy of this license, visit http://creativecommons.org/licenses/by/4.0/.

(C) The Author(s) 2020 